\title{
La lengua guaraní dependiente en tiempos de Independencia en Paraguay
}

\section{Bartomeu Melià (S. J.)}

\section{(2) OpenEdition}

\section{Journals}

Edición electrónica

URL: https://journals.openedition.org/jsa/11904

DOI: 10.4000/jsa.11904

ISSN: 1957-7842

Editor

Société des américanistes

\section{Edición impresa}

Fecha de publicación: 20 diciembre 2011

Paginación: 153-174

ISSN: 0037-9174

\section{Referencia electrónica}

Bartomeu Melià (S. J.), «La lengua guaraní dependiente en tiempos de Independencia en Paraguay», Journal de la Société des américanistes [En línea], 97-2 | 2011, Publicado el 10 diciembre 2014, consultado el 02 septiembre 2022. URL: http://journals.openedition.org/jsa/11904 ; DOI: https:// doi.org/10.4000/jsa.11904 


\title{
LA LENGUA GUARANÍ DEPENDIENTE EN TIEMPOS DE INDEPENDENCIA EN PARAGUAY
}

\author{
Bartomeu MELIÀ, S. J. *
}

Desde el siglo XVII, pero sobre todo en el siglo XVIII, la lengua guaraní desarrolló una abundante literatura dentro de los pueblos o reducciones guaraní-jesuíticas del Paraguay. Algunos escritos en lengua guaraní fueron publicados en esa época, pero una gran parte de ellos son manuscritos que se conservan hasta hoy en archivos y bibliotecas. Esa literatura no trata sólo de temas religiosos - catecismos, sermones, libros de piedad -, sino que son expresión de la vida política, social y cultural de los guaraníes. Son en realidad escritos no sólo en guaraní sino de guaraníes. Este tipo de documentación va más allá del tiempo de presencia de los jesuitas en los pueblos, de los que se les hizo salir en 1768, de manera que se puede hablar de guaraní jesuítico sin jesuitas. Por extraña paradoja la documentación en guaraní se interrumpe después de proclamarse la independencia del Paraguay en 1811, a pesar de que el uso del guaraní siguió siendo el medio de expresión de todo el pueblo paraguayo, como lo atestiguan diversos viajeros que visitaron el Paraguay a lo largo del siglo XIX. [Palabras claves: Paraguay, independencia política, lengua guaraní, corpus histórico, política lingüística.]

La dépendance de la langue guarani à l'époque de l'Indépendance au Paraguay. Dès le $\mathrm{XVII}^{\mathrm{e}}$ siècle, mais surtout au cours du $\mathrm{XVIII}^{\mathrm{e}}$ siècle, une importante littérature guarani a vu le jour dans les villages - ou réductions - guarani-jésuites du Paraguay. Quelques écrits en guarani furent alors publiés, mais beaucoup d'entre eux sont demeurés sous la forme de manuscrits aujourd'hui conservés dans des archives et des bibliothèques. Cette littérature ne traite pas seulement de sujets religieux (catéchismes, sermons, livres de piété); elle témoigne aussi de la vie publique, sociale et culturelle des Guarani. Ces textes ne sont pas seulement en guarani, mais de Guarani. Cette documentation déborde de l'époque où les jésuites résidaient dans les villages dont ils furent expulsés en 1768: on peut donc parler d'un guarani jésuite sans jésuites. Paradoxe étonnant, cette documentation en guarani se tarit après la proclamation de l'Indépendance du Paraguay en 1811 alors même que le guarani demeure la langue relationnelle de tout le peuple paraguayen, comme l'attestent plusieurs voyageurs qui visitèrent le pays au $\mathrm{XIX}^{\mathrm{e}}$ siècle. [Mots-clés: Paraguay, Indépendance, langue guarani, catalogue historique, politique linguistique.]

* Río Apa 2100, B Trinidad, Casilla de correo 3290, Asunción, Paraguay [bmelial@hotmail.com]. Journal de la Société des Américanistes, 2011, 97-2, pp. 153-174. @ Société des Américanistes. 
The submission of the Guarani language in the aftermath of Paraguayan independence. From the 17th century on, with a peak in the 18th century, vast amounts of literature written in Guaraní language were produced in the Jesuit-Guaraní settlements or Reductions of Paraguay. Some of the texts were published back then, but many remain in manuscript form in archives or libraries. This literature does not only deal with religious matters (catechisms, sermons, or devotional books). It also reflects the political, social and cultural life of the Guaraní people. In fact, these documents are not only written in Guaraní, but also written by Guaraní, and they continued to surface even after the Jesuits had been expelled from the settlements in 1768. Jesuitic Guaraní therefore seems to have outlived the Jesuits' actual presence. By some strange paradox, after the proclamation of Paraguayan independence in 1811, Guaraní texts were no longer found, even though Paraguayans went on communicating in Guaraní language on a daily basis, as reported by numerous travellers who visited the country in the 19th century. [Key words: Paraguay, political independence, Guaraní language, historical corpus, linguistic politics.]

Ni las revoluciones más radicales ni los cambios políticos más acentuados logran cambiar automática o repentinamente la lengua de la sociedad donde esos fenómenos históricos se dan ${ }^{1}$. Para el cuadro lingüístico del Paraguay, la fecha concreta - digamos el 14 de mayo de 1811 - es irrelevante. Por supuesto, la lengua - las lenguas - que hablaba el día antes de la Independencia la siguiera hablando el día después. La lengua como fenómeno social sólo manifestará sus cambios a largo plazo, si bien es cierto que puede transformarse con mayor rapidez en determinadas circunstancias y con la aplicación de políticas lingüísticas definidas.

Ahora bien: ¿Cuál era la lengua de los habitantes de esta tierra en el período - no en el momento-de la Independencia? Delimitemos convencionalmente este período entre 1760 y 1840. En 1760 estaban todavía los jesuitas en sus 33 pueblos o reducciones donde el guaraní era lengua propia y única; en 1840 ocurre la muerte del supremo dictador, José Gaspar Rodríguez de Francia, que ha excluido sistemáticamente al guaraní en la gestión oficial de su gobierno. En estos años, se han dado dos hechos de consecuencias relevantes. Primero, el extrañamiento de los jesuitas de todos los dominios de la Corona española y, concretamente, de los pueblos guaraníes del Paraguay, decretado en 1767, pero llevado a cabo solamente en julio y agosto de 1768, ya que, por razones de orden práctico, no era posible en menos tiempo encontrarles sustitutos en otras órdenes religiosas - franciscanos, dominicos o mercedarios. Y ahí viene una segunda cuestión: ¿La independencia política en Paraguay tuvo alguna repercusión en la vida lingüística?

Algunos países, cuando se constituyen en estados independientes, marcan su independencia mediante una ruptura con la lengua de estado dominador anterior. Sin embargo, en la immensa zona que hoy llamamos América latina 
ninguna independencia introdujo un cambio de comportamiento ni de usos lingüísticos. A pesar de que se difundieran algunas proclamas y decretos en lenguas amerindias, se siguió con la lengua castellana, considerada lengua propia de los mismos independentistas y lengua oficial sin discusión (Levene 1947-1948). Y las lenguas indígenas todavía habladas mantendrían el mismo estado y situación, permitidas pero no apoyadas por la nueva realidad política.

El Paraguay no sería la excepción. Ahora bien, esto es lo extraño, que este país haya seguido la regla general, a pesar de que el castellano no fuese la lengua usual y propia de la nueva nación.

\section{EL CORPUS DEL GUARANÍ EN LOS TIEMPOS DE LA INDEPENDENCIA}

Los documentos del período considerado que aleatoriamente han llegado a nuestras manos provienen de archivos formados casualmente, donde los papeles en guaraní vienen mezclados generalmente con otros en castellano, a veces agrupados cronológicamente o por asunto.

La mayor parte de los documentos del siglo XVIII han sido conservados en el Archivo General de la Nación Argentina (AGN) en Buenos Aires, pero no se tiene todavía un catálogo completo de ellos, ni explicación de su contenido. El arquitecto e historiador Norberto Levinton ha localizado una buena cantidad de ellos. Gracias a su correspondencia personal he tenido acceso a un gran número de ellos. En la tesis doctoral de Neumann (2005, pp. 363-381) se indica también, no siempre de forma precisa, una larga « lista de documentos indígenas (guaraní misionero) ». Son 172 en total los citados, muchos sólo en guaraní, mientras que de una buena parte sólo se ha conservado una versión castellana y, de otros, tanto el texto original guaraní (o su copia) como el castellano, que ciertas autoridades requerían.

También en el Archivo Nacional de Asunción (ANA) se tiene una rica serie de esos manuscritos que provienen de los pueblos adscritos al Paraguay. Son papeles de especial interés que muestran fehacientemente la continuidad del guaraní jesuítico después de los jesuitas, pero también su evolución hacia formas de escritura y de lengua cada vez menos estandarizadas y vulgares. Merece una especial atención el codicilo de la Biblioteca del Museo Mitre que contiene, entre otros muchos papeles de los pueblos guaraní-jesuíticos en lengua guaraní, las « Proclamas del general Belgrano » a las autoridades y pueblo del Paraguay. Éste es en realidad el más importante documento político de la época, en cuanto a expresión de un pensamiento político moderno y nuevo en una lengua americana, como no sucederá ya más. El contenido original de los textos hay que atribuirlo al mismo Belgrano, pero la versión en guaraní que hace ese secretario del pueblo de Candelaria, muy probablemente originario del lugar y ciertamente un buen y 
aventajado discípulo de la escuela y tradición jesuítica, hace de él un traductor y un pensador de la nueva realidad política que se anuncia.

Hay otras piezas guardadas en diversos acervos casuales. Hay documentos que ya fueron publicados, sobre todo en su versión castellana. Los editores que se atrevieron a una transcripción del texto guaraní, incurrieron por lo general en graves errores, que desfiguran y a veces hacen incomprensible el documento. En el apéndice I está un inventario incompleto de los documentos más importantes y significativos de ese período, por orden cronológico.

\section{ESPLENDOR Y DECADENCIA DEL GUARANÍ (1760-1810)}

De hecho, los cabildos de los pueblos misioneros escribían sus actas en guaraní y de esta suerte dirigían también su correspondencia a las autoridades españolas. Los numerosos papeles conservados atestiguan el uso general de la lengua por los cabildos de los pueblos y por particulares. Durante la llamada guerra guaranítica entre 1750 y 1756, volaban los correos por los pueblos, incluyendo simples billetes. Un conjunto de siete cartas llegaron a Buenos Aires y el gobernador las entregó al padre Juan Delgado, rector del colegio de los jesuitas, para que las tradujese del guaraní al castellano, quien lo hizo con la mayor fidelidad, palabra por palabra, con una prolijidad poco amena y nada imitable, dirá otro jesuita. Esas cartas que manifiestan visión política, sinceridad y coraje extraordinarios, se conservan en el Archivo Histórico Nacional, en Madrid (AHN, leg. 120j). En una Relación ( $\mathrm{n}^{\mathrm{o}} 7$ ) incluida en ese legajo consta que « los caciques, sin que lo sepamos - dice el padre Luis Charlet -, y sin hacer caso de nosotros, envían su gente, se avisan con sus papeles de día y de noche ». La práctica de la comunicación escrita no era pues algo excepcional entre los guaraníes de los pueblos.

Entre las fechas que hemos seleccionado para nuestra atención, 1760-1840, la literatura en guaraní muestra diversas facetas, sobre todo después de la salida de los jesuitas, ya que puede hablarse de guaraní jesuítico sin jesuitas.

En cuanto al contenido, el bloque que gira alrededor de la expulsión de los jesuitas, que afectó especialmente a los pueblos en donde los padres atendían en lo espiritual, pero también supervisaban muy de cerca la administración temporal, expresará durante años las quejas de los indios frente a una medida para ellos inconcebible e injusta, si bien un grupo de corregidores y caciques halagados y seducidos con regalos y honras ridículas por las autoridades españolas dirán que ahora se sienten libres de ellos y un nuevo modo de vida será posible.

Después del extrañamiento de los jesuitas, y en relación implícita con el estado anterior, se manifiestan otros problemas, derivados generalmente de la nueva administración. Ahí exponen su sentir sobre los tributos exigidos por la 
Corona española, se quejan de los « españoles » que han entrado en sus pueblos, dan cuenta del cambio de autoridades, hacen pedidos y reclamaciones. En algunos pliegos, anotan el movimiento de entradas y salidas de mercancías de sus almacenes. Las piezas que provienen de los antiguos pueblos de los que los jesuitas no hacía mucho habían sido expulsados, presentan una caligrafía más elegante y una ortografía más coherente, muy agradable de ver. Son pequeñas obras de arte que, por desgracia, degenerarán a medida que se acercan al siglo xix. Con el paso de los años, caligrafía y ortografía se empobrecen.

Los documentos que consideramos no sólo permiten estudiar el guaraní de la época - una tarea que se está abordando en la actualidad -, sino también el castellano circunstancial de esta parte de América, con sus particularidades, su curiosa ortografía y las capacidades de los traductores frente a textos que muchas veces superaban sus competencias. Esos textos, al mismo tiempo, dan cuenta de la realidad del Paraguay, donde la sociedad es de una u otra lengua, siendo los verdaderamente bilingües más bien raros y escasos.

De entre esos documentos, no se puede eludir la Carta del pueblo de San Luis, de 1768 (British Museum, Add. 32605, p. 23102, f. 37r-39r.), de la que transcribimos aquí algunos párrafos y en la que se conjugan la reverencia y obediencia a la autoridad con una opinión muy crítica sobre la situación creada, junto con una sabia ingenuidad, que dice las mayores verdades sin ambages. Humildad, enojo, ironía y firme coraje están magistralmente reflejados en la carta, que es ocasión para los autores de protestar por el extrañamiento y salida de sus « queridos padres », decretada por Carlos III.

Dios te guarde a ti que eres nuestro padre, te decimos nosotros, el Cabildo y todos los caciques, con los indios e indias y niños del pueblo de San Luis.

El Corregidor Santiago Pindó y D. Pantaleón Cayuarí con el amor que nos profesan, nos han escrito pidiéndonos ciertos pájaros que desean enviemos al Rey. Sentimos mucho no podérselos enviar, porque dichos pájaros viven en las selvas donde Dios los crió, y huyen volando de nosotros, de modo que no podemos darles alcance. Sin que eso obste, nosotros somos súbditos de Dios y de nuestro Rey, y estamos siempre deseosos de complacerle en lo que nos ordene; habiendo ido tres veces a la Colonia como auxiliares, y trabajando para pagar el tributo, y pidiendo como pedimos ahora que Dios envíe la más hermosa de las aves, que es el Espíritu Santo, a ti y a nuestro Rey para iluminaros y que os proteja el Santo Ángel.

Ah, señor Gobernador, con las lágrimas en los ojos te pedimos humildemente dejes a los santos Padres de la Compañía, hijos de San Ignacio... Esto pedimos con lágrimas todo el pueblo, indios, indias, niños y muchachas, y con más especialidad todos los pobres. [...]

Los Padres de la Compañía de Jesús saben conllevarnos, y con ellos somos felices...

Además, que nosotros no somos esclavos, ni tampoco gustamos del uso de los españoles, los cuales trabajan cada uno para sí, en lugar de ayudarse uno a otro en sus trabajos de cada día. [...] De San Luis, a 28 de Febrero de 1768.

Tus pobres hijos, a saber, el pueblo y Cabildo entero. [Siguen las firmas] 
No estaba el horno para bollos, y no están los indios para loros.

Como muestra de la problemática en los años posteriores a la expulsión de los jesuitas está la larga Carta del Cabildo de Santa María de Fe, de 1788 (ANA, SH, vol. $152, \mathrm{n}^{\circ} 5$, fol. 1-2). A las tres páginas del texto guaraní se adjunta una síntesis en castellano de la misma época, que es la que transcribimos aquí [ver MEC, Archivo Nacional de Asunción, Documentos en guaraní (1770-1850), Asunción 2004, 14-16]. Quien pueda interesarse por el texto guaraní lo encontrará en el apéndice II.

Sor. Gov. ${ }^{\mathrm{r}}$ Intt. ${ }^{\mathrm{e}}$ y Cap. ${ }^{\mathrm{n}}$ gral.

El protector de los naturales de esta provincia con la debida atención, dice que el Corregidor y Cabildo del pueblo de Santa María de Misiones expone a V.S. con mucha sumisión en el anterior memorial, que con motivo de que se van acrecentando sus ganados ya le son perjudiciales los muchos pobladores arrendatarios que hay en sus terrenos, especialmente Don José de Espínola, Don Gabriel Duarte y Don Antonio Toñanes por estar estos muy cerca de las estancias del mismo pueblo, y por esta causa se hallan todos mezclados y de aquí es, y nace el perjuicio: Por lo que ocurren a la piedad de V.S. se sirva tomar providencia a fin de que siendo cierto, mandar que estos despueblen y todo los demás que sean perjudiciales, por el bien del pueblo por cuya causa que es una de las legítimas pruebas el dueño expulsar al locatario arrendatario o inquilino y es prevención legal que cuando el dueño necesita la finca para su habitación, o cuando el inquilino le es incómodo, puede cobrarla sin embargo de cualquiera contrato de alquiler, o arrendamiento. Y sobre todo, V.S. se servirá determinar lo que sea más arreglado a justicia. Asump. ${ }^{\mathrm{n}}$, y septiembre 19. de 1788. [firmado] Juan Bap ${ }^{\text {ta }}$ de Achard

Muy cercano a la fecha de la Independencia es el expediente promovido por el corregidor y el cabildo del pueblo de Santa Rosa de Lima contra el actual administrador Tomás Esperati, fechado en 1808 [ANA, SNE, 2900, fol. 118-120 vuelta, 1808; ver Documentos en guaraní (1770-1850), 40-45]. La versión en castellano es casi de la misma fecha (ANA, SNE, 2900, fol. 122-126 vuelta, 1808), el texto guaraní original lo encontrará el interesado en el apéndice III.

Señor Subdelegado $=$ El Corregidor y Cabildo del pueblo de Santa Rosa ante VM. con toda veneración nos presentamos haciéndole saber lo que ha sucedido en este nuestro pueblo con nuestro mayordomo Don Tomás Esperati; éste lo dejó de mayordomo en este pueblo el Señor Gobernador Intendente para que cuidase de todo en primer lugar el cuidado de la iglesia y en segundo para que atendiera al aumento del fondo de propios asignándole de gratificación el 10 por ciento; pero no obstante esto no ha cumplido con su obligación dicho Esperati y menos con lo que se le ha mandado no saliendo a ver ni acompañarnos en los trabajos de afuera tampoco ha salido a reparto de terrenos ni de yeguas no atendiendo en nada al adelantamiento de propios ni menos hace con todo lo demás que hemos trabajado y trabajamos en el año pasado y en este año el diariamente nos dice que todo lo paga pero, como queda dicho no hemos visto que entre en el almacén un valor, pues tan solo el valor de unas misas ha sido lo que ha entrado en el almacén. 
Dónde está el valor real paño celeste que de nuestro almacén compró nuestro cura y dónde está cinco pesos que el mismo cura entregó al mayordomo valor de una sepultura a la que deberá de contestar el mayordomo, pues lo que únicamente hemos visto entrar en el almacén son veinticinco pesos que entregó el padre cura valor del paso también contestara que se ha echo como todo el mes de diciembre pasado viéndose obligado nuestro Corregidor en vista del poco cuidado del mayordomo a buscar cebo para hacer hubiese otra vez sacramento fuese alumbrado no obstante había en nuestro almacén bastante lienzo y plata para comprar lo necesario para la iglesia, pero sin p. a el no se descuida de comprar lo necesario de donde sacamos en consecuencia que para cuidar de sí y su bien está únicamente obtien esta mayordomía de este pueblo de Santa Rosa también hasta ahora no sabemos el paradero de tanto trigo que tuvimos abundantes de libertad pues todo el solo lo hace aprovechando ni al padre cura tampoco les ha dado dando únicamente de este trigo y cincuenta vara que con el nombre que era para Santa Rosa se tejió de esta pieza cortó el mayordomo 30 varas y los restantes se lo entregó al padre cura para que lo vendiere a cuatro reales la vara cuyo provecho entregó otro padre al mayordomo que ascendió a ciento y un pesos de este dinero solo se ha gastado para la procesión de la patrona ocho pesos que se dieron a los revestidos de la misa y lo restante no sabemos en qué se habrá invertido también nos manda componer un algodonal con el pretexto que su producto se había de gastar en la función del pueblo lo que tampoco hemos visto pues en dicho día solo se nos repartió un poquito de miel, sal y unos cuantos mazos de tabaco de pito, no sabemos donde estará el aguardiente y chocolate todo esto nos hace este mayordomo por ver que somos unos pobres engañándonos como mejor le parece pues lo contrario hemos visto también responderá al cargo de los 14 pesos que mensualmente paga don Francisco Soler por razón de comida y casa pues no hemos visto entrar en almacén este enero, también deberá contestar sobre lo que paga en maestro Platero y el español Chaparro, también aclarará el asunto de las 41 cabezas de ganado vacuno de uno a dos años que trajo don Dionisio González Villaverde, de este ganado dijo el mayordomo que iba a tomarlo para gasto lo que así se lo dijo al casero, pero lo que hizo fue sacar vacas gordas... Quedan referidas por estos motivos faltan telas para la iglesia, también deberá conquistar al cargo de los cortes de carretas y doscientas varas de tablas que de esto debe ser sabedor el maestro carpintero Juan Gregorio Chaparro y de treinta una silla en armajes, pues nada de esto ni su valor ha entrado en el almacén ni menos constan en los diarios de este mayordomo también responderá al cargo de las herramientas que tiene el mayordomo a su servicio pues nada sabemos ni vemos entrar en el almacén; también responderá el mayordomo a cargo que le hacemos de las mulas que se llevó don Dionisio Caballero, suponemos sería con licencia de VM., señor esta saca; todo esto está en los primeros 5 meses que se dio la orden por el señor gobernador intendente para la suministración de raciones que en ella se detallaban tanto efecto y después ahora ya no; el 20 de marzo último reconvino el corregidor al mayordomo el motivo de no dar las referidas raciones a lo que contestó el mayordomo que no quería que se mataran dos reses respondiendo alterado que no temía al señor gobernador ni a nadies escandalizándonos con estas expresiones también responderá que con que licencia tiene ocupados a los panaderos tahona y caballos del pueblo sin se sepa si paga o no sus conchabos y alquileres como también las vacas lecheras que tiene pues son del pueblo, de las... suele echar manos de ellas para este fin como lo ha hecho con 22 bueyes todo esto ha sucedido desde nos 
libertamos lo cual nos tiene muy descontento por lo que damos a V.M., parte bajo de juramento a fin de que se sirva poner remedio según lo que exige el caso y lo exponemos en esta representación que ponemos en manos de V.M. en la que pedimos justicia y en prueba y fuerza de ello lo firmamos en este pueblo de

31 de marzo de 1808

Porque no saben firmar: Ignacio Tyi Secretario de Cabildo.

Está traducida según su tenor que antecede. Candelaria 7 de abril de 1808. Antonio.

Candelaria 21 de abril de 1808

Devuelvan esta presentación y traducción al señor subdelegado del departamento de Santiago. Lovato

En este pueblo de Santiago a los 26 días del mes de abril de 1808 yo el subdelegado de este departamento en atención a lo que representa el corregidor y cabildo del pueblo de Santa Rosa en el escrito que hace cabeza y resultando de su traducción al idioma español que enseguida obra garantizado por don Antonio Morales intérpretes del superior gobierno de esta provincia.

Estos tres documentos muestran la situación de unos pueblos que van evolucionando hacia una escritura pragmática de comunicación no tanto interna sino con un mundo exterior, también colonial, pero que se les ha vuelto hostil y ajeno. La sociedad guaraní de los pueblos jesuíticos no estaba enteramente alfabetizada y lo prueba el hecho de que muchos de sus dirigentes no saben ni siquiera firmar, pero hay una clase de secretarios indígenas que alcanzan grados de excelencia escrituraria notable. El pueblo paraguayo tardará todavía dos siglos para recuperar un nivel de comunicación escrita semejante. Y si no mantuvo su calidad de lengua guaraní, tampoco avanzó en el conocimiento del castellano, que en los dos siglos después de la Independencia siguió enclenque.

\section{Las proclamas del general Belgrano al pueblo paraguayo (1810)}

No hemos encontrado papeles de claro contenido político para esa época en el Paraguay, pero los hay que provienen de fuera. Cuando el general Manuel Belgrano en 1810, en vísperas de la Independencia, dirigía proclamas y cartas en guaraní a las autoridades del Paraguay y a su pueblo, lo hacía a sabiendas de que ésta era su lengua propia. Como anota certeramente Morínigo (1968, p. 198), «escribirlas en guaraní no constituía un gesto de político habilidoso para halagar sentimientos regionalistas. Era simplemente una actitud realista impuesta por una necesidad concreta. El pueblo paraguayo, como el correntino y misionero, por una serie de circunstancias históricas, sólo conocía la lengua indígena ».

Las piezas que constan en el códice que se conserva en el Museo Mitre de Buenos Aires, son sin duda copia de los papeles realmente enviados o por enviarse - a este respecto tengo mis dudas. Los textos fueron probablemente redactados 
en el pueblo de Candelaria, antigua reducción, sede del superior jesuita de las Misiones del Paraná hasta la fecha del extrañamiento en 1768. En Candelaria fueron ciertamente traducidos o, mejor dicho, adaptados al guaraní. La cuidada y delicada caligrafía, así como la correcta y coherente ortografía dan fe de que el copista fue un antiguo secretario del pueblo o cercano discípulo de los pendolistas del tiempo misionero. Los trazos y estilo de letra lo denotan claramente al ser comparados con otros documentos de la época.

El códice consta de 22 páginas y se presenta en dos columnas, castellano y guaraní. El primer escrito viene bajo el título de Oficio.

Las llamadas «proclamas » del general Manuel Belgrano a autoridades y pueblo del Paraguay, que constan en el codicilo de la Biblioteca del Museo Mitre, copias manuscritas a dos columnas de los textos en guaraní y castellano son los siguientes:

1 Kuatia ombohasa va'ekue Exmo. señor Oficio del Exmo. Sr. D. Manuel don Manuel Belgrano, mburuvicha Belgrano, vocal representante de la Paraguáipegua upe don Bernardo de Exma. Junta Gubernativa de las Velasco. 6 de diciembre de 1810

Provincias del Río de la Plata y General en Jefe del Ejército del Norte pasado al Sr. Gobernador Intendente del Paraguay Fernando de Velasco. 6 de diciembre de 1810

2 Ambuae kuatia imbohasapyre Cabildo Paraguaipegua upe.

Para el cabildo de Asunción.

6 de diciembre de 1810

3 Poromorandu [Paraguáipegua upe].

Proclama [a los paraguayos].

4 Kuatia imbohasapyre obispo Paraguáipegua upe.

5 Poromarandu [Misiones táva ñavo ñavõyua...]

6 Ambue imbohasapyre Don Pablo Tompson upe. 6 de diciembre de 1810.

7 Comandante Itapua upegua kuatia imbohasapy.

Ambue imbohasapyã Comandante Candelaria táva rováipe õ̃ va'e. 17 de diciembre de 1810.

Al obispo del Paraguay. 6 de diciembre de 1810.

A los naturales de los pueblos de Misiones.

Al comandante don Pablo Thompson.

Al comandante de Itapúa, 17 de diciembre de 1810 .

Al comandante del frente de

Candelaria, don Tomás Mármol. 17 de diciembre de 1810.

9 Poromorandu general ojapo va'ekue

Al propio ejército de operaciones.

A modo de ilustración copiamos la proclama a los pueblos de Misiones, en su texto castellano y en su versión al guaraní, versión decimos porque se aleja mucho de una traducción propiamente dicha del texto suministrado por Belgrano. Es un texto más conciso, menos ampuloso y más directo. 


\section{PROCLAMA}

Naturales de los pueblos de Misiones: la excelentísima Junta provisional gubernativa, que a nombre de su majestad el señor don Fernando VII rige las Provincias del Río de la Plata, me manda a restituiros vuestros derechos de libertad, propiedad y seguridad de que habéis estado privados por tantas generaciones, sirviendo como esclavos a los que han tratado únicamente de enriquecerse a costa de vuestros sudores y aun de vuestra propia sangre: al efecto me ha nombrado su representante, me ha revestido de todas sus facultades y a puesto a mi mando un ejército respetable: ya estoy en vuestro territorio, y pronto á daros las pruebas más relevantes de la sabia providencia de la excelentísima Junta, para que se os repute como hermanos nuestros, y con cuyo motivo las compañías de vosotros que antes militaban en el ejército entre las castas, por disposición de nuestros opresores, hoy están entre los regimientos de patricios y arribeños; pedid lo que quisiéredes, manifestándome vuestro estado, que sin perder instantes contraeré mi atención a protegeros y favoreceros, conforme á las intenciones de la excelentísima Junta; pero guardaos de faltar al respeto debido á sus justos y arreglados mandatos y de contribuir á las sugestiones de los enemigos de la patria y del rey; pues así como trabajaré por vuestra utilidad y provecho, si cumpliereis con vuestras obligaciones, del mismo modo descargaré la espada de la justicia sobre vosotros, si, olvidados de lo que debéis á la patria, al rey a vosotros mismos siguiereis las huellas de esos mandatarios, que sólo tratan de la ruina de estos fieles y leales dominios del amado Fernando VII, y de cuantos hemos tenido la fortuna de nacer en ellos. Manuel Belgrano.

De este original sale la siguiente versión en guaraní, aquí transliterada en ortografía actualizada:

\section{POROMOARANDU}

Misiones táva ñavõ ñavõygu, tapeikuaa Exma Junta ko ára ñande jokuái vae Ñande Réi Don Fernando $7^{\circ}$ rérape, che mbou pene mö̈vo teko aguyjeípe, ako livre pendekoháva amokãngatávo, aguijéma niko, pene renonde guare heguive peiko eguï pende jokuaihára tembiguáiramo, pende ry'aikuépe ha'e penduguýpe oñemoingo aguijevae kue; aiporehe Exma Junta che mbou guekovia etéramo, ha'e opu'aka rusuháva reheve aru che ndive kapaju eta. Ä̈ma pende yvýpe, añekuave'ë peme mboriahu apireýpe, ha'e ahechaukáne peme [peëme] ako Exma, Junta jesarekoháva, opopytyvõne, ha'e apombo'eteukáne, ha'e ahechaukáne ore amo pende rekoháva, ha'e imboupyguávo tapeikuaa kapaju eta pendeýi ojehúma ko ára imbojehe'ápyramo ore pa'üme, ha'e oikóma ipeapýramo eguï heko guejyve vae pa'ü hegui omoï hague hegui eguï ñande jopyhare: pejerure peipota vae tanime añeha'áne opopytyvövo Exma. Junta jesareko jave, ha'e aete ani ave pe'atoipota, iporokuaita mboajevo, ani ave peñeha'ä pota pe poropytyvõvo ñande Réi, ha'e ñande moangekóiha ñande yvýpe; añekuave'ë porä jave peëme, aikuave'ë ave Justicia po'aka rusu opomboarakua haguä, pende resaráiramo guarä Ñande Réi ñande yvy, ha'e pendejehegui pe poropytyvöse potávo eguï ñande jokuáire ha ey ngatu háre ãva niko, oñeha'ã vae a yvy Fernando $7^{\circ}$ ñande rembiayhu potávo ave. Manuel Belgrano.

La expedición de Belgrano al Paraguay fracasó militarmente, aunque tuvo efectos ideológicos que se hicieron sentir en poco tiempo. Es cierto que podemos dudar de que sus proclamas en guaraní hayan sido leídas por las personas a 
quienes iban dirigidas, y aun de que hayan llegado realmente a sus destinatarios, pero son el testimonio de alguien que quiere llegar al paraguayo y sabe que lo tiene que hacer en su lengua, que en la época es el guaraní. La Independencia del Paraguay en 1811 no fue ajena a los principios formulados por Belgrano que, por otra parte, se propagaban desde varios núcleos de conciencia política.

La iniciativa de Belgrano no era por otra parte del todo inédita, ya que en la misma época se hacía uso de otras lenguas indígenas, como el quechua y el aymara, para proclamar las ideas del movimiento emancipador (Levene 1947-1948).

\section{LA LENGUA GUARANÍ SUMERGIDA (1811-1840)}

Según todos los testimonios de propios y extraños que se refieren al período en el que se da nuestra Independencia, la lengua propia y ordinaria del Paraguay era el guaraní. Y sin embargo, los padres de la Independencia parecen ignorar el hecho. No encontramos un solo documento de carácter político - o de Estado que se exprese en guaraní.

Entre los documentos conservados en el Archivo Nacional - según lo que por ahora conocemos - hay uno de 1813, y viene de un tal José Antonio Aripuy de Candelaria, que denuncia las vejaciones del comandante de la plaza, Vicente Antonio Matiaúda (AN, SCyJ, 1385, 1, f. 1-2). El otro es ya mucho más tardío, de 1850 y, por lo tanto, del tiempo de Carlos Antonio López. Se trata de la causa criminal del esclavo Blas, por proferir palabras insultantes contra el gobierno. El documento está en castellano, pero las palabras « tan insolentes » del pardo o mulato, con licencia de su excelencia, por lo groseras, se reproducen en su auténtica expresión guaraní: " ko tacho aña ocorre ñane retãme, ndaiporivéi ningún comercio ko ãga ñandéve ko aña ra'y monda este gobierno ãgagua ogoberna guive ñane retã ». Es un testimonio del guaraní popular, de la calle, que será usual hasta los días de hoy. Después, el registro oficial de la vida en guaraní desaparece, desaparece incluso en los pueblos de misiones que, a partir de 1848, fueron declarados ciudadanos comunes. Al mismo tiempo que la lengua escrita, esos pueblos ven sus tierras cada vez más invadidas por los nuevos estancieros cuyos nombres iban apareciendo desde años atrás en los documentos de denuncia.

En la larga dictadura del doctor Gaspar Rodríguez de Francia (1814-1840), si hubo envíos de documentos en guaraní desde los pueblos o por particulares, no fueron dignos de ser archivados.

\section{El guaraní AUSENTE}

¿Cómo explicar entones la ausencia casi total del guaraní como lengua « oficial » en y después de la Independencia del Paraguay en 1811? 
La Independencia fue realizada ante todo por la oligarquía criolla terrateniente y militar, y ésta conocía por lo general la lengua guaraní. La gente paraguaya de los años de la Independencia hablaba más guaraní incluso que en los tiempos coloniales inmediatamente anteriores. El hecho es que al llegar al poder, el doctor Gaspar Rodríguez de Francia, dictador supremo entre 1814 y 1840, suprimió el Real Colegio Seminario de San Carlos, en 1822. La secularización de todas las órdenes religiosas afectó también muy de cerca a las posibles élites culturales. Se puede afirmar que, a partir de la década de 1820-1830, solamente es representativa del Paraguay la denominada gente rei, es decir, « el pueblo común », según el análisis histórico de R. E. Velázquez. Los grupos sociales con capacidad e interés por emplear la lengua castellana, como podía ser la burguesía comercial y la oligarquía terrateniente, heredera de encomenderos y « comuneros »- menos éstos que aquellos - fueron reducidos a callarse, cultural y políticamente (Granda 1988, p. 544).

Se puede decir que el Paraguay independiente es entonces más guaraní que español y ciertamente menos bilingüe, si es que alguna vez lo ha sido. Los testimonios sobre el uso de la lengua guaraní como única lengua de la región se repiten constantemente, como ha anotado Morínigo (1968, pp. 202-203).

Los hermanos Robertson, que habían visto al gobierno del Dr. Francia como un « reino de terror », ofrecen al mismo tiempo no pocos detalles de una vida cotidiana apacible y hasta feliz, pautada por fiestas y conversaciones nada sofisticadas. "Conversación - en guaraní - era la mejor manera que tenían hombres y mujeres en el Paraguay para pasar sus noches » (Robertson 1839, en Nagy 1969, p. 101). Los mismos registran que « el pequeño Orrego, cuando su pulpería estaba llena de gente baja arengaba con elocuentes tiradas en guaraní en elogio del Caraí Francia ».

El guaraní se re-oralizó con la Independencia; los manuscritos del período muestran que el guaraní va perdiendo su status de lengua escrita, y se hace cada vez más vulgar, pudiéndose dar a esta palabra vulgar el sentido de lengua del vulgo y lengua inculta.

\section{La DEPENDENCIA DEL CASTELlano}

Con la muerte de El Supremo, en 1840, y aunque se insinuara enseguida, por parte de sus sucesores, una crítica a la política cultural anterior y una preocupación por castellanizar al país a través de la instrucción pública, la situación lingüística del Paraguay no iba a cambiar tan pronto. Y son de nuevo los viajeros y comerciantes los que aluden a la lengua guaraní como hecho social peculiar del Paraguay. Los testimonios son constantes y unánimes.

Es corriente en el campo que las personas entiendan y hablen sólo el guaraní con excepción de los que son funcionarios públicos o han recibido alguna educación [...] 
Aunque parezca extraño, y a pesar de que el padre (Don Juan Bautista Rivarola) es, para este país, un hombre instruido que estuvo a punto de ser nombrado presidente, ni su mujer ni sus hijas hablaban el español. (Munck 1843-1869, en Nagy 1969, p. 192)

La lengua que se habla en el Paraguay es el «guaraní », que hablaban los indios que habitaban el país en la época anterior a la llegada de los españoles. Este idioma es de uso tan general que nunca se habla el español, y sólo con los extranjeros, siendo, en el interior, la gran masa de la población tan ignorante del español que es necesario tener un intérprete incluso para pedir un vaso de agua. Entre las mejores familias, en las ciudades y pueblos mayores, se comprende el español, y se lo habla con los forasteros; sin embargo, hasta en Asunción hay gente que no lo conoce en absoluto. Los decretos y leyes del gobierno se publican en español, que se usa también para dar órdenes en el ejército, y se lo enseña en las escuelas, a las cuales todos los niños varones del país deben ir forzosamente, hasta que aprendan a leer y escribir. Mas después de dejar la escuela, quizás nunca más lo escuchen, pues no tienen libros para leer; hay muy poco papel para escribir de vez en cuando, con la excepción de firmar sus nombres y, generalmente, antes de cumplir 21 años, ya olvidaron todo lo que aprendieron en la escuela. (Graham 1846, en Nagy 1969, pp. 138-139)

Un diplomático francés, Eugenio Guillemot, ponía de relieve un aspecto curioso, por lo demás discutible, de la lengua guaraní paraguaya, al desvincularla de una hipotética herencia racial. «Los habitantes de estas campañas hablan solamente el guaraní pero su fisonomía no es americana; los hombres tienen aspecto de europeos y las mujeres, vestidas con trajes asiáticos, llevan con mucha gracia sobre la cabeza o al hombro elegantes vasijas de tierra roja » (Guillemot 1857, en Nagy 1969, p. 179).

El empleo del guaraní para el chiste y el piropo, que todavía suscita orgullo entre los paraguayos, fue entendido por un entusiasta inglés que veía en el Paraguay una romántica Arcadia, y que se animó a aprender guaraní y usarlo no sin gracia.

Estoy aprendiendo también el guaraní que, siendo una rara jerigonza, sin embargo, no carece de elegancia. Está lleno de vocales, mas la mayor dificultad consiste en que las consonantes se pronuncian sin diferenciarlas, de manera que resulta difícil representarlas fonéticamente. La palabra guaraní que significa agua, es el punto crítico de la lengua: los paraguayos dicen que ningún extranjero es capaz de pronunciarla. [...] Todos los paraguayos, varones y mujeres, hablan guaraní y la mayoría de las clases bajas no hablan otro idioma; sin embargo, el idioma oficial es el español y la gente de sociedad afecta despreciar el guaraní [...]. (Mansfield 1856, en Nagy 1969, pp. 205-206)

Un médico y naturalista francés, Alfred Demersay, en sus investigaciones filológicas sobre la lengua guaraní (1859), da cuenta de ciertas particularidades y funciones sociales de la lengua guaraní dignas de tenerse en cuenta. «En el Paraguay el tuteo es general, y esta costumbre, tan contraria a las fórmulas consagradas por la bella lengua castellana, explicaría hasta cierto punto la 
ausencia de desigualdad social que ahí se observa » (Demersay 1859, en Nagy 1969, p. 150).

A través de estos testimonios que cubren los primeros años de Independencia, no se nota que la situación lingüística del país haya sufrido cambios sensibles, pudiéndose observar pautas comunitarias en el uso de las lenguas castellana y guaraní, que se gestaron en el período colonial y que serán válidas hasta el siglo XX.

El guaraní es, evidentemente, la auténtica lengua del Paraguay, hablada por todos sus habitantes sin excepción. El castellano, por el contrario, es código lingüístico aprendido « a puros azotes » (Cardiel) en las escuelas. Su conocimiento y utilización está limitado a una minoría caracterizada por las siguientes notas: localización preferentemente urbana (Cardiel, Graham, Munck), sexo masculino (Cardiel, Dobrizhoffer, Robertson, Munck), edad adulta (Cardiel, Dobrizhoffer) y posición social elevada unida a nivel cultural alto (Azara, Munck), aunque, incluso en estos casos, el guaraní es la lengua de uso primario, manejada hasta por «el encomendero y su familia (Cardiel)». (Granda 1988, p. 541)

La paradoja consiste en que, después de medio siglo de independencia, tenemos un Estado lingüísticamente castellanizado en un pueblo enteramente guaranizado. *

* Manuscrit reçu en mai 2010, accepté pour publication en février 2011.

\section{Note}

1. Texto basado en la conferencia que se iba a dar en el V Congreso Internacional de la Lengua Española, 2 al 5 de marzo 2010, Valparaíso, Chile y se anuló por motivos del terremoto. Una versión preliminar de este texto se encuentra en http://www.congresodelalengua.cl/programacion/seccion_i/ melia_bartomeu.htm.

\section{REFERENCIAS CITADAS}

Brabo Francisco Javier

1872 Colección de documentos relativos a la expulsión de los jesuitas de la República Argentina y del Paraguay en el reinado de Carlos III, Estab. y tip. J. M. Pérez, Madrid, pp 277-289.

Catañeda Carlos E. y Jack A. Dabbs

1952 Calendar of the Manuel Gondra manuscript collection, Editorial Jus, México.

GraNDA Germán de

1988 Sociedad, historia y lengua en el Paraguay, Instituto Caro y Cuervo, Bogotá.

HERNÁNDEZ Pablo

1908 El extrañamiento de los jesuitas del Río de la Plata y de las misiones del Paraguay por decreto de Carlos III, V. Suárez, Madrid. 
LEVENE Ricardo

1947-1948 «Las revoluciones indígenas y las versiones a idiomas de los naturales de documentos de la Independencia », Boletín de la Academia Nacional de Historia, 20-21, pp. 81-90, Buenos Aires.

Melià Bartomeu

2003 La lengua guarani en el Paraguay colonial, Cepag, Asunción.

Morínigo Marcos A.

1968 «Para la historia del español en la Argentina. Las cartas guaraníes del general Belgrano », Actas de la Quinta Asamblea interuniversitaria de Filología y Literaturas hispánicas, Universidad Nacional del Sur/Instituto de Humanidades, Bahía Blanca, pp. 197-204.

NaGy Arturo

1969 Paraguay: imagen romántica (1811-1853), Edic. Centenario, Asunción.

Neumann Eduardo Santo

2005 Prácticas letradas guaraní: produçãoe usos da escrita indígena (séculos XVII e ${ }_{X V I I I}$ ), tese de doutorado, Historia Social, Universidade Federal de Río de Janeiro, Río de Janeiro.

PÉREZ-Cuesta Luis

1996 Documenta iesuitica, año II (5-6) [s. n.], Praha, República Checa.

Rivet Paul y Georges De CRÉQui-MontFort

1951 Bibliographie des langues aymará et kičua. I. 1540-1875, Institut d'ethnologie, París [reproducción facsímil de un volante, que trae el documento en cuatro lenguas: español, kícua, aymará y guaraní, pp. 244-248].

SUSNIK Branislava

1966 El indio colonial del Paraguay, II. Los trece Pueblos guaranies de las Misiones (1767-1803), Museo Etnográfico Andrés Barbero, Asunción.

\section{ApÉndice I}

\section{Lista parcial de documentos en guaraní entre 1761 y 1845}

[En el Museo Mitre, Sección Archivo, Documentos coloniales. Arm B.C.18, P. 27, nº de orden 32, existen 25 documentos de diversos pueblos de las misiones, en los que las autoridades guaraníes informan sobre el cumplimiento de una orden del gobernador Pedro de Cevallos, probablemente del 21 ó 22 de marzo de 1761, para formar compañías de soldados en vistas a la defensa de los territorios del Uruguay. Estos oficios, en castellano y/o en guaraní se extienden entre el 24 de abril y el 20 de mayo de 1761 (ver Pérez-Cuesta 1996)]

1758-1785 «Colección de documentos en idioma guaraní correspondientes a los Cabildos de indígenas de las misiones jesuíticas del Uruguay desde el año 1758 al 1785. Manuscritos ", Biblioteca Museo Mitre [BMM] (Melià 2003, p. 365) [Algunos de los documentos de esta colección vienen aquí desglosados conforme a la sucesión cronológica]. 
1761 Oficio dirigido por el pueblo de Santa María la Mayor, respondiendo a una orden de aprontar tropas y armas para enfrentar a los portugueses, 8 de abril, BMM.

Carta al gobernador de Buenos Aires, Pedro de Zevallos, Trinidad, 14 de abril, BMM.

Oficio dirigido por el pueblo de Santos Mártires, sobre lo mismo, 16 de abril, BMM.

Oficio dirigido por el pueblo de La Cruz, sobre lo mismo, 25 de abril, BMM, dos oficios.

Oficio dirigido por el pueblo de Encarnación, sobre lo mismo, 1 de mayo, BMM.

Oficio dirigido por el pueblo de San Ignacio Guasú, sobre lo mismo, 9 de mayo, BMM.

Oficio dirigido por el pueblo de Nuestra Señora de Fe, sobre lo mismo, 12 de mayo, BMM.

1766 Lista de 78 indios de San Boja y Santo Tomé que acompañaron a un resguardo, solicitados por Martín de Altolaguirre, en 7 de julio, BMM.

1768 Carta de Juan Antonio Curigua al gobernador don Francisco Bucareli, 4 de marzo, AGN, Sala IX, 6-10-7.

Billete del teniente don Valentín Ybarygua, de San Miguel, a los de Yapeyú, 25 de abril, AGN, Sala IX, 3-3-4.

Carta de los Treinta Pueblos, Archivo Nacional de Chile, Fondo Jesuitas, vol. 159.

Carta de Don Valentín Ybarygua al gobernador D. Pedro Cevallos, en que agradece el envío de nuevos Padres, San Miguel, 12 de agosto, AGN, Sala IX, 6-10-7.

Carta de Don Valentín Ybarygua al señor gobernador D. Francisco Bucareli, en que pide licencia para obtener caballos, San Miguel, 13 de agosto, AGN, Sala IX, 6-10-7.

Billete de Cristóbal Arira, agradeciendo favores recibidos por él y su familia, Itapua, 10 agosto, AGN, Sala IX, 6-10-7.

Billete del teniente don Valentín Ybarygua, de San Miguel, al gobernador, 13 octubre, AGN, Sala IX, 6-10-7.

Carta de Narciso Guyrabo al gobernador, Yapeyú, 27 de agosto, AGN, Sala IX, 6-10-7.

Declaraciones de dos Yndios del Pueblo de San Ygnacio Guazú que presentaron un Papel, repugnando la recepción del Cura y Compañero que se han destinado para aquel Pueblo, y pidiendo les dejasen al Jesuita que se salía de él. Año de 1768, BM, Add. 32605. p. 23102, f. 83r-85r, en Melià 2003, p. 365.

Carta en lengua guaraní, en la que el pueblo de San Luis ruega que permanezcan entre ellos los Padres de la Compañía de Jesús. 28 de febrero 1768, British Museum, Add. 32605. p. 23102, f. 37r-39r (publicada en Hernández 1908, pp. 364-369. Transcrita con muchos errores en Woodbine Parish, Buenos Aires y las Provincias del Río de la Plata desde su 
descubrimiento y conquista por los Españoles, 2 tomos, Buenos Aires, 1852. Copia en la Biblioteca del Museo Mitre, en Melià 2003, p. 365).

Carta del Gobernador de Buenos Aires al Conde de Aranda, remitiéndole adjunta otra en idioma guaraní (con su traducción castellana), escrita a S. M. por los corregidores y caciques de treinta pueblos, situados entre los ríos Uruguay y Paraná (publicada en Brabo 1872).

Carta de Diego Guacuyú al gobernador Francisco de Paula Bucareli, Yapeyú, 26 de septiembre 1768, AGN, Sala IX, 6-10-7.

Carta del cacique Chrisanto Tayuare al gobernador Francisco de Paula Bucareli, Yapeyú, 18 de septiembre 1768, AGN, Sala IX, 6-10-7.

1770 Lista de géneros y cantidades a ser distribuidas a los caciques, Candelaria, 20 de abril 1770, AGN, Sala IX, 17.4.5, Guar.

Declaración o reconocimiento de corregidor del pueblo de San Joaquín, ANA, SH, 55,17, f. $7 \mathrm{v}$.

Carta al Sr. Administrador general Julio Gregorio Espinosa de varios cabildantes; secretario Faustino Yoritu, AGN, Sala IX, 17.4.5.

Carta de los caciques del pueblo de Loreto a D. Francisco de Paula Bucareli, gobernador de Buenos Aires. Loreto, 17 diciembre, AGN, IX-17.4.2, en Susnik 1966, pp. 27-28 y Melià 2003, p. 365.

Recibo de entrega de caballos, Candelaria, 11 de agosto, AGN, Sala IX, 18.6.6, Guar./Cast.

Recibo de la hacienda al pueblo de Apóstoles de géneros embarcados a Buenos Aires, Candelaria, 14 de marzo, AGN, Sala IX, 18.5.2, Guar./Cast.

Recibo del procurador de Santos Mártires por venta de 20 novillos, Candelaria, 23 de marzo, AGN, Sala IX, 7-7-7, Guar./Cast.

1772 Carta al Sr. Administrador general D. Juan Angel Lezcano. Corpus, 20 de mayo, Biblioteca del Museo Mitre.

Carta del cabildo, Corpus, 18 de mayo, AGN, Sala IX, 18-6-6.

Carta a D. Francisco Bruno de Zavala, gobernador de la provincia del Paraná y Uruguay, San Juan, 13 de octubre, AGN, Sala IX, 22.2.7.

1773 Certificado del cabildo de Santos Mártires a favor de de Juan Matheo Martínez, Santos Mártires, 28 de abril, AGN, Sala IX, 18.6.6.

1776 Carta al Teniente Gobernador Don Juan Valiente de Cabildos y Caciques de San José, noviembre de 1776, AGN, Sala IX, 17-6-3.

Copia de dos cartas en lengua guaraní de la estancia de San Nicolás, en las que se dice de los « intentos de los enemigos y las persuasiones que hacen a estos Indios para separarlos de la obediencia al rey », 4 de abril, remitidas por Francisco Bruno de Zavala. AGN, Sala IX, 17-6-1.

1779 Carta del Cabildo del pueblo de Yuty al Padre Visitador para que se les envíe religiosos doctrineros, 24 de octubre, TxU, MG 1620. 3 f. r-v (f. 19-21), en Castañeda y Dabbs 1952, p. 206 y Melià 2003, p. 365.

Orden que el padre Miguel Martínez traduzca una carta del guaraní al español, en Castañeda y Dabbs 1952, p. 206.

1780 Carta al Virrey de Buenos Aires de los tripulantes de un "itapa » de madera (jangada) en que piden indemnización por pérdida de vestuario, AGN, Sala IX, 17.7.1, en Susnik 1966, p. 214 y Melià 2003, p. 366. 
1783 Carta del corregidor y cabildo del pueblo de San Estanislao, al gobernador Melo de Portugal, ANA, SNE, 227, f. 28.

Carta del corregidor y cabildo del pueblo de San Joaquín, al gobernador Melo de Portugal, ANA, SNE, 227, f. 31.

1784 Carta del pueblo de Altos, 16 de marzo, BM, Add. 27, 601, f. 250-253, en Melià 2003, p. 366.

Representación del Cabildo del pueblo de los Altos pidiendo que se deje al Cura de administrador, 15 de marzo, BM, Add. 27, 601, f. 246-249, en Melià 2003, p. 366.

1785 Carta de Diego Cayure, Santa María Mayor, 7 de octubre, AGN, Sala IX, 27-1-1.

1787 Carta de indios del pueblo de Santa María de Fe en que se pide la libertad de algunos presos dirigida al gobernador D. Joaquín de Alós, sin fecha [1787?], en Melià 2003, p. 366.

$1788 \quad$ Carta del Cabildo del pueblo de Santa María de Fe al señor gobernador Alós contra estancieros arrendatarios establecidos en sus tierras, ANA, SH, 152, 5, f. 1-2, 1788, en Susnik 1966, pp. 196-198 y Melià 2003, p. 366.

Estado de cuentas sobre ganados y otros bienes administrativos de los pueblos jesuíticos del Paraná, ANA, SNE, 218, f. 108.

1789 Carta del corregidor Hermenegildo Curuguao a don Francisco Silvero, administrador del puesto de San Joaquin, ANA, SNE, 600, f. 85.

Cartas de varios Cabildos de Yndios Guaranís, de algunos de sus individuos, y Curas de sus respectivos Pueblos que manifiestan el júbilo, y dan gracias por la variación de su gobierno opresivo en Comunidad y por otras Providencias particulares del Exmo S. ${ }^{\text {or }}$ Marqués de Avilés, siendo virrey de Buenos Aires.

1792 Presentación de nómina de cabildantes del pueblo de Caazapá, ANA, SNE, 525, f. 8.

Pueblo de la Santísima Trinidad, ANA, SNE, 3370, f. 91.

1803 Carta de María Aripuy y su hija Rosa Guaraparé, al corregidor y cabildo del pueblo de Corpus, ANA, SCyJ, 1388, 1, f. 63.

1808 Expediente promovido por el corregidor del cabildo y cacique del pueblo de Santa Rosa de Lima, contra el actual administrador, Tomás Esperatti, ANA, SNE, 2900, f. 118.

1810 «Proclamas de Belgrano » (ver detalle supra).

1811 Circular./José Ramón Yrá Administrador de la Renta del Estado, Representante y Corregidor de la villa de San Miguel, San Carlos, del Departamento del Paraná, al Norte, en Colección de datos y documentos referentes a las Misiones como parte integrante del territorio de la Provincia de Corrientes, Corrientes, 1877, pp. 193-196.

1813 Decreto Junta gubernativa 1811. Extinción del tributo, en Paul Rivet y Georges de Crequi-Montfort, Bibliographie des langues aymará et kičua. I, Institut d'ethnologie, París, 1951 [reproducción facsímil de un volante, que trae el documento en cuatro lenguas: español, kíčua, aymará y guaraní], pp. 244-248.

José Antonio Aripuy contra el comandante de Candelaria, Vicente Antonio Matiauda, sobre vejaciones, ANA, SCyJ, 1385, 1, f. 1-2. 


\section{APÉNDICE II}

Carta del Cabildo de Santa María de Fe (SH. vol. 152, n ${ }^{\circ}$ 5, fol. 1-2, 1788, Documentos en guarani 1770-1850, 14-16).

Ore ruvicha Guasu Al Señor Gobernador. Señores por todo Los Cavildo

Tuvicha vichave niko nemomirĩ guasu pype oroñembojapávo nde ypyguýri oroñemoĩmbávo

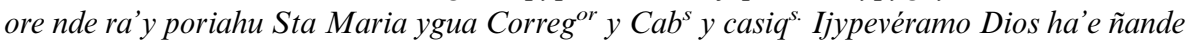
Rey Guasu Corona réra pype ha'e nde mbojeroviávo ave ãga Señor Gov ${ }^{[o r]}$. Ápe niko oro'evo ãga ndéve Tupã rera marangatu ha'e katu ome'ẽ haguã ndéve tekove marane'̃̃ ha'e katu nde resape haguãma.

Opa nde rembiaporã pavẽ pype ikatu va'e nde erejapo haguãma ha'e ikatu eỹ va'e nde rejapo ẽ̃ haguãma erereko nde pope ãga Sr. Gov ${ }^{r}$. Kova'e hupigua oro'e ãga ndéve, Señor, porque ndéramo ererekóramo cargo tuvicha ete va'e ore opa katu rehe ãga, Sr., Dios ha'e ñande Rey Don Carlos Tercero parokuaitáva rupi ha'e ipy'a marangatuháva ha'e iporoporiahu vereko guasu háva ave ijavegua e'ỹ va'e pype nde ave erereko ãga nde jehe nde py'a marangatuháva pype ãga, Sr. Kova'e rehe ãga niko. oroguereko pavẽ jerovia guasu ete

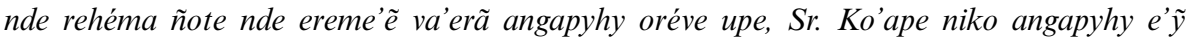
oroguereko va'e pype niko, Sr.

Chate pánga rako ãga oroguereko va'e angapyhyei ko ore yvy mirĩ poriahu ore estancia pype. Opa ore rymba vaka ha'e yeguas oroguerekopa ãga, Sr. Ha'e a'ete na ore rymba kuéra meme ruguãi. Aipóramo ã va'e karai poblador rymba kuéra ave oikévo ave ore rymba kuéra pa'üme. Kova'e rupi ojehekáramo [ojeherraramo] ojehe'aháva rehe, ojehapývo tava rymbáramo ha'e kova'e ohecha riréramo, ohecha icapata kuéra kórupi omboja contramarca hese oikuaáramo oñangarekoháramo heko; ha'e rire ambue oime ivaive va'e ore ra'y estanciero ñemokane'ôháva oñemoĩramo yeguas recohida ojese'apa guasuháva rehe poblador rymbakuéra rehe ave, ndoikuaáirihávare. Akoi tape omondoseha koty ambuae rymba iñaka hatã oipysývo tape ambua'e ñote ã va'e rehe ave oñemokane'õvo ore ra'y estanciero poriahu, Sr. Gobernador. Kova'e rehe ore huvicha kuéra oroikuaa jepe angã oroguerokirirĩ ñote angã. Ha'e ko árape opa orombojekuaapa ndéve, Sr., ha'e nde katu ikatuha rapicha ndéve ha'e oréve ereme'ẽ haguã angapyhy. Chate pãnga rako oĩ va'e ore rymba katúhape ha'e ore rymba kuéra jejapihápe oĩ va'e Don José Espinola, ha'e rire Don Gabriel Duarte ha'e rire Don Antonio Toñánes. Kova'e Don Josef Casar [Casal] niko oguereko capatas aguyjei ombo'aje porãgueko ha'e okaraiñe'ẽ imboaje porãvo ha'e oũ porã ñote ore rymba kuéra vahẽ ỹhápe. Kova'e hupigua oro'e'ndéve, Sr. Governador.

Ha'éramo ko'ãga orombojekuaa va'e ave ãga ndéve, Señor, ou ovahẽ ore estancia po pype oroguereko va'e puesto ore ra'y romoĩ hápe, he'ivo ore ra'ýpe: kova'e tendaguy eresyrine ha'e arúne ápe, Sr. Obipo rymba ojávo. Kova'e rehe ore kuéra correg ${ }^{r}$ y cav ${ }^{o s}$ y caciques oroikuaa porã e'ỹvo jepe ãga, Sr. Ndouíri ramo jepe nde hegui porandu oréve ãga orohendúramo tuvicha etei va'éramo hekoháva rehe. Kova'e rehéramo hupigua hekóramo nde ereme’ẽ ymáramo licencia chupe ore ave orome'ẽ ore rorypápe ñote ãga, Sr. Kova'e rupi toñembo'e ave Tupã upe ore angareko katuráma ha'ekane'õ kuéra ore karu haguã hovasama haguãma rehe. 
Kova'e ñote ãga ave oro'e ndéve, Sr. Kova'e ijajéramo, ndoroipotavéi eguĩ ore rymba karuha popy rupi oĩ va'e Sr. Espinola; ha'e rako he'i va'ekue oréve upe ãga che rymba aru jepe ko pe yvyýpe ha a'ete pehechakua ymáramo pe rymba kuéra oñemboheta peẽme; che momarandu ñote he'i va'ekue oréve ha'e, aipe'apa jevýne che rymba kuéra, ojávo oréve. Aipo rire kova'e Sr. Duarte ave oroipota osyry haguã ha'e ave, togueraha guymba kuéra mamo ha'e omo'ãrohápe; ha'éramo topyta ñote hupigua hekóramo ou hague pa'i Cabayero Sr. Obispo rérape ha'e nde rérape ou haguéra rehe, Sr. Governador.

Kova'e ñote oro'e ãga ndéve ko ore kuatia mirĩ poriahu oromoĩnde pópe, Sr. Gov ${ }^{r}$, ha'e ãga katu oroñemoímbávo nde pyguýri ñemomirĩ ete pype nde po pysývo ave ãga, Sr. Ijypýramo Dios ha'e ñande Rey Don Carlos Tercero rérape enéi ãga, Sr. Dios katu topyta nde pýry ha ta nde resape ave nde py'a marãngatuháva toirümo ngatu ndéve. Ko rupi ereme’ẽ núne angapyhy oréve ko oro'e va'é upe ndéve. Ko rupi jareko haguã angapyhy ko yvy ári ha'e rire uku tupã retãme ñande manõramo jahupity haguãma tory vusu opa'ẽ̃ va'eráma jepiguáramo.

Amén

Eupe hina oho orehegui pavẽ ko ore Katia nde pópe imox́a'e $S^{r}$. Gov ${ }^{r}$

Alferez Real Christ. ${ }^{\text {l Guyrapepy }}$

Regidor Segundo Pasqual Jucuy

y Casiq. ${ }^{\text {es }}$ Dn Juan Bautista Guyrapepy

Casiq. ${ }^{e}$ por el Correg.r D.n Miqel Ñandutii

Por el Cav.o que no saben firmar Ignacio Cherembi

Caziq ${ }^{e}$ su secret. ${ }^{\circ}$ de Cav. ${ }^{\circ}$ Don Basil. ${ }^{\circ}$ Quarepy

Assum. ${ }^{n}, 19$ de septiem. De 1788.

Para el Protector de Naturales para que se presente en forma cuando hallare por conven. ${ }^{\text {te }}$ Alós

\section{ApÉndice III}

Expediente promovido por el Corregidor, Cabildo y caciques de Santa Rosa de Lima (ANA, SNE, 2900, f. 118, 1808, Documentos en guarani 1770-1850, 40-45).

Señor Sub delegado

Ore ra'ýramo Tupã remimoingue nde ñangarekoháramo ko Santa Rosa de Lima táva rehegua Corregidor ha'e Cabildo opa katu, oroñemoĩvo nde pyguyri ñemomiringatu pype oroi kuaaukávo ndéve ko ore táva poriahúpe ijaie va'e ko'ára rupi, ko ore karai Mayordomo Don Tomás Esperati héra va'e oheja va'e kue niko Señor Gobernador Intendente ore Mayordomo ko Santa Rosa távape, opa mba'e rehe oñangareko va'e ramo, tenondeve ramo ore Tupao rehe iñangareko haguã oñeme'ẽ va'ekue chupe kuaitáva aiporire táva propio rehegua mba'ete tiro rehe, ha'e avae rupi ogana haguã diez por ciento okane'ongue repyramo ha'e ha'e aete nombo ajeiri o obligación oñemenda va'ekue ojeupe ha'e noseiri mamove ohecha haguã ako ore rembiapo noremoiruĩvo mamove rupi, ni yvy mboja'o hárupi, ni yeguas mboja'o ha rupi ndohechái oiko eỹvo ore yrũ ramo ndoiporaraivo kuarahy, ha'e kane'õ ore yru ramo, ni ha'e ndoi ko kuaai kavaju áramo, ndoguerekói py'a jejuka mba'eve táva Propio ramo rehe, noremongetáivo araka'evei tarea rekorã amo rehe ko tera Estancia mo rekorã rehe, ku peina ore estancia vaka mbovy õ̃ va'e opa potaima omomba orehegui, evokói ramojepe ndohecha kuaai mba'eve hekovia ramo rehe ore moinge tae ymo, tove mante ha'e tokaru rei hina para eso apaga he'i mante pero aete ndorohechái mba'eve hepy oike va'e Almacenpe, ko so'o 
hembi'u kue rehe, ha'e grasa, ha'e sebo hacienda oguereko va'e Tupao rera pypegua, ha'e ñote ojesape ipype, opa ã mba'e ha'e ogusta oĩma, ha'evo koi remombota ore pa'i cura Santísimo ocunsumi va'ekue peteĩ jasy diciembre guetevo oroĩ sacramento eĩ rehe va'ekue akói ramo, opa ãvae rechavo rako ore ruvicha Corregidor Seno omono'õ hekomi omoĩ uka jevy haguã Santísimo Sacramento oreve, ha'e aete ku ore mayordomo nopenái mba'eve rehe oĩme hápe ore kane'õngue almasénpe lienzo ha'e plata, ha'e rirepã chupe guarã ndoatai ha'e Ñandejára upe guarã oata okoi ramo ha'e ojeupe guara mba'e rehe ñote opy'a ambohasývo, ha'eko ijepy'a mbohasýva aguyjema so'o, ñandy, ha'e kure kyra mante kovae rante nipo oiko ore mayordomo ramo, ko ore táva Santa Rosape, ha'e rire ko trigo ore ra'y reta poriahu kane'ongue oroje liberta ẽ̃ moveramo trigo egui tetá ambue kue rupi iporu pyre rekovia he rupire mamo nipo avae oĩme, ndoro hechái, ha'eño oaprovechapávo ã mba'e, ni Pa'i Abare kuéra, ni avave yrũ ramo oaprovecha eỹvo, aguyjéma Pa'i Abare ohecha va'e Ñandejára rete ramo misa marangatúpe mante ha'e rumive kuehe ro'y opavaepe ha'e rami aveite, ha'e anga gua ha'eramiveme, opa avae ore kane'onngue repy ndorohechái hamiko oréve apaga apaga ojávo ha ore ndorohechái aipova'e araka'evei oike va'e almacen pe trigo repy aguyjema mburika repy uvei orohecha oike va'e almasen pe, kupe agui katu mba'eve repy ndorohechái, ha'e kóina ore kuaa ogueno va'ekue paño celeste, kova'e repykue viñaete ndoro hechái, ha'e kóina cinco pesos plata sepultura repy pa'i cura ome'ẽ va'ekue ichupe ndorohechái ave, ipaha ore rembiechakue veinticinco p. plata Pa'i omoinge va'e almacen pe paso oiporu hague repy, ha'e kóina una pieza lienzo Santa Rosa rérape oje japo va'ekue docientas y cincuenta varas va'ekue, ha'e kovae hegui oikytĩ vie kue ha'e treinta [ü] ha'e hembyrekue ogue ro'y kue uka ore Pa'i cura kotype, ha ovendeuka a cuatro [rr] varape, ha'e ojapo ore cura peteĩ ciento un pesos - Plata, ha ome'êko ore mayordomo upe ha'e avae hegui ojegasta patrona árape pa'i omisa purahéi va'ekue upe ojepaga cuatro pesos ha mokõive revestido upe a dos pesos cada uno, ha'e kovae rembyrekue marã nipo oiko ore ndoroikuaavéima kovae he'i va'ekue niko oréve ko petẽ mandyjuty pe moatyrõ pende ára ramo, con eso tapereko mba'e pegasta va'erã pe patrona árape pende pohupape guarama ojávo oréve aipo he'i rire aguyjéma eira mirĩ, ha'e juky mirĩ ña'ẽ apu'a mirĩme, ka'a mirĩ, ha'e petỹ de pito [mase] peteĩ teĩ avae mante orohecha va'ekue akói ramo, mamonipa oĩme oréve imombe'u pyre aguardiente, cominos, pimienta, clavo, canela, ha'e chocolate avae niko mboriahu ore rekópe orembotavy avaepe akói ramo orere rekove oñembosarai hárama ha'e kóina karai Francisco Sole viñae ko ore távape oĩ ypyguive koty oiporu hague, okaru hague opagava jasyñavo catorce pesos plata pype, ãvae viñae ndoro hechái oike almacen pe kupei cha avei nipo opaga egui karai mokõi platero, ha'e karai Chaparro, opa avae niko ndoro hechai he' i kue almacen pe kóina hina karai Dionisio González Villaverde heravae ogueru va'ekue Estero gui cuarenta y una vaka ra'y de dos años ha'e uno rehe guave, kovae viñae he'i cabildo upe oipyhy haguã gasto ramo, ha kovae repy ha'e oguenohe vaka guyra katupyry, opa ãvae remimbota oata ore tupao pe tataendy Ñandejára peguarã, opa arami niko orereko ñembosarái háramo ava poriahu orerekópe, kóina hina ore ra'y reta poriahu kane'õngue ako oroje liberta eĩmove guare, ha'e oroje liberta marire mokõi karreta rangue maso, ha'e [vagason] ha'e tabla peterevy guigua, ha'e cedro guiguave como doscientas ũ péina hina peteĩ español Juan Gregorio Chaparro hera vae maestro carpintero ha'e mante nipo oikua e guivae reko, ha'e treinta y una sillas armasen hacienda ramo ijapo pyre, opa avae repy ndoigueiri almacen pe ndorohechái opa avae niko mba' eguipa nomoingéi va'ekue o diario pe ko karai Mayordomo ra'e, ore ndoroikuairi egui va'e mba'e ha eupeina hina herramientas azada y servicio pe ojeporuva'e ore oroikua eiva'e opa avae repy ore ndoroikuaari almacen pe ndoro hechái ha'e kóina hinave karai Dionisio Cavallero upe oguerahauka va'ekue mulas nde 
licencisa pe nipo ra'e señor kovae ave ore ndoroikuaari avei, hepykue oike hague almacen ko ara peve ndori kuaai, opa amba'eniko ojejapo ko ore táva poriahupe, kóina hina nde poroporiahuvereko rupi erejerure va'ekue señor gobernador upe ore pa'i kuéra upe ikarurã mirĩ so'o, jepe'a mirĩ, ha'e y, opa ãvae nde kuaaitáva niko oromboajevora'e señor, opa maestro, sacristán, ha'e mba'e asývo poriahupe oñeme'ẽ haguã so'o mirĩ, kovae niko nde porokuaitéva cinco veces pe mante ijaie porã va'ekue akói ramo ha akói guive ndijajeveima ko nde poro kuaitague anga peve, pa'i Avareupi oiko so'o ñembo harui hague ime'êmbyramo, mbyte ã mboriahu vete retaupe akói ára guive noñeme'ẽ veivo ración mirĩ chupe kuéra, aguyje kuehe Corregidor 20 de marzope ojapo cargo chupe ko mboriahu kuéra maestro, ha'e sacristán upe oñeme'ẽvei ha ración mirĩ maray charamopa oñeme'ẽvei ha chupekuera kova'e niko Señor Gobernador porokuaitague, pero aete ha'e he'i va'ekue che ndaipotái mokõi veces ojejuka ojavo Corregidor ha'e Cabildo upe, che ndakyhyjéi avave gui, ni Señor Gobernador guio javo, opa ãmba'epe oñe henduka pochy oréve ha'e ore mbo'e katupyry harãngue ohechauka oreve, hendukavo mal ejemplo, opagueko, ha'e guembiapope, ha'e rire péina hina ore ra'y poriahu mbujape opoha kuera opa aramemepe ombae apo va'e chupe nigue koteverã amo apo eỹmo, omboaje potavo ikuaitáva, ha'eko [Cavaju] hacienda tahoero, opa avae mavae licencia pe pa ra'e ãnga peve oipo ruherekovo, ha'e ramive lechera o kamby uvae, opa ãvae ore ndoroi kuari, avapa ome'ẽ licencia chupe ra'e, aiporamo jepe ndipóri ramo gasto ojukarika lechera mansa hacienda imemby kuera reheve ramo mbovy supimanipo ho'u ra'e ha'e ramive Buey Hacienda veinte y dos y tantos rupima ore oroikuaava'e, kovae niko akoi ro'y oroje liberta ypy guive orohecháva, ha'e oroi kuaa va'e, ha'e oroguero anga pyhy ẽ̃ va'e. Anga oromombe'uvo ndéve ko'yte ko juramento pype py'a marangatu hape ñote, na ako iñamotareĩ hápe ruguái aete, nahese ma'e pochy hape ruguai ave, hi'angavae ramo heko pe ñote, ha'e rami ore ave ore anga rehe oromonde hape ñote oroi kuaauka katupyryvo ndéve ko arapype.

Aiporamo rehe nia oromoivo ko ore Presentación ndo pope ko ára, ha nde hegui katu oroguereko jevy hagua ãngapyhy ko'yte, ha'e korami pype mavaeve amo oma'ẽ ỹ haguã ko nde justicia rehe, kovae hupigua ete heko hápe ramo oromoĩvo orefirma chupe ymo kanata haramo. Dios tome'ẽ anga ndéve rekove marane'ỹngatu ára heta rupi vyteri ore pave upeguarama [1] marzo de 1808.

Francisco Xavier Poy Corregidor

Gregorio Arapi Alcalde $2^{\circ}$ [voto]

Miguel Mboresay Alf $R 1^{\circ}$

Por mí y los demás del Cabildo que no saben firmar

Ignacio Tyirie Secretario de Cabildo. 